\title{
Automated Bias-Correction for Accurate FCD Processing Systems
}

\author{
Günter Kuhns, Rüdiger Ebendt, Elmar Brockfeld, and Wolfgang Niebel \\ German Aerospace Center, \\ 12489 Berlin, Germany \\ \{Guenter.Kuhns, Ruediger.Ebendt, Elmar.Brockfeld, Wolfgang.Niebel\}@dlr.de
}

\begin{abstract}
Within the European research project SimpleFleet, a fleet management system for small and medium enterprises has been developed. GPS traces are collected from equipped vehicles and fed into an FCD processing system to generate traffic data. To estimate the quality of derived traffic data and determine the systematic bias of a FCD processing system a previous method called "self-evaluation" was applied for specific times of day and roads with similar characteristics. With the assumption that the relative bias is the same for corresponding traffic situations, it can be transferred and used to correct results of further computations. Notice that this opens up a convenient road for automated online corrections based on really large amounts of data, rather than on small data sets from costly measurement campaigns.
\end{abstract}

Keywords: Floating Car Data (FCD), Correction of Bias, Quality of Traffic Data

\section{Introduction}

Real-time traffic information is one of the most important data for operative traffic management as well as for traffic-related applications. In the European research project SimpleFleet with the main application areas geomarketing and fleet management systems for small and medium enterprises, the cost efficient access to these data with coverage of a whole road network were important requirements. Since conventional sources did not meet these requirements and equipped logistic fleets can also be used as sensors to generate further traffic data, FCD was chosen as source for this information. While other systems store information that allows identification of individual drivers, FCD only requires the re-identification of a vehicle during one trip and allows collection of traffic data while respecting the privacy of its users.

During the last years the Institute of Transportation Systems at the German Aerospace Center (DLR-TS) has developed algorithms and technologies to exploit GPS data from probe vehicles (“floating cars”). Especially taxi fleets have been used in several applications as probe vehicles realizing implementations for different cities in Europe and Asia. Current work is mostly focused on the handling of new sources for traffic data with similar characteristics (e.g. smartphones with GPS sensors), creating new fields of FCD applications, assessing and improving the quality of 
generated FCD. For a number of different reasons, FCD processing systems can be prone to systematic bias, e.g. because of the need to decompose the travel time between two subsequent reporting positions to several links [5], and because of GPS measurement errors, due to clouding or multi path signals.

In many investigations to assess the quality of FCD, measurement campaigns were conducted, using vehicles which are equipped with higher quality GPS systems [1],[2],[3]. While these campaigns yield reliable results that can be evaluated and compared to FCD results easily, they are quite costly and usually only cover short times or small areas.

\section{Self-Evaluation Approach}

The self-evaluation approach presented here relies on the basic assumption that the observed actual travel times for individual vehicle trajectories can be used as a ground truth for the mean link travel times computed by a FCD system.

If this assumption holds, then, for a particular observation period, the absolute systematic bias can be computed as the difference of two mean values, namely the mean actual trajectory travel time and the mean travel time on these trajectories computed by the FCD system (see [4]). More precisely, the first mean value is that of $m$ observed actual travel times $o_{i}$ for $i=1, \ldots, m$ for individual vehicle trajectories (denoted $\bar{o}$ ), and the second mean value is that of the travel times $\mathrm{c}_{\mathrm{i}} i=1, \ldots, m$ computed by the FCD system along the same trajectories at the time of observation (denoted $\bar{c}$ ). The travel times $c_{i}$ for $i=1, \ldots, m$ are computed by summing up the mean link travel times computed by the FCD system at the respective periods of travel on a trajectory, for all links constituting the respective individual trajectories.

It is of note that, in the scope of self-evaluation, the mean link travel times are computed without use of the link travel times observed for the vehicle which generated the respective trajectory. In other words, yet it is computed as usual as the arithmetic mean of the travel times of all individual vehicles observed on that link during the respective period, but the link travel time of the vehicle which drove the ground truth trajectory is excluded from this arithmetic mean. This is done in order to avoid any circular reasoning, which would be introduced by comparing an observation, namely the actual trajectory travel time, with a computed value (partly) based on exactly this observation.

The relative systematic bias then of course is the ratio of the absolute systematic bias and the mean observed actual trajectory travel time, given as percentage $\frac{\overline{\mathrm{c}}-\overline{\mathrm{o}}}{\overline{\mathrm{o}}} *$ $100 \%$. Also notice that the original method as described in [4] yields only one global value for the overall systematic bias of the FCD system per observation period (e.g., one hour), and that only one data source (i.e., one vehicle fleet) is considered. 


\subsection{A First Extension of the Approach: Analysis with Additional Separation Criteria}

A practical implementation can use a digital road map. In such a map, links of the road network are usually tagged with constructional attributes like e.g. speed limits. A first extension of the self-evaluation approach makes use of this fact to obtain a more fine-granular distinction of the data used for determining systematic bias.

Already this first extension (like the final approach presented in this paper) is also based on the following basic assumption: it is assumed that on all links with identical constructional attributes, there are similar traffic conditions for corresponding periods. Moreover, one assumes that similar relative systematic biases are in effect on links with identical constructional attributes in corresponding periods. Then, no further distinction needs to be made between such links, and, for every set of corresponding periods, the same relative systematic bias can be assumed and later applied for correction.

In a first extension of the existing algorithm, five hierarchical street categories from 0 (highways and other similar major roads) to 4 (minor roads) used by the provider (Navteq/HERE) of the digital map to tag street segments, were used as separation criterion. For each category $j=0, \ldots, 4$ the sums $\bar{o}_{j}$ and $\bar{c}_{j}$ are generated separately as $\bar{o}_{j}=\sum_{i=1}^{m} o_{j i}$ and $\bar{c}_{j}=\sum_{i=1}^{m} c_{j i}$ with $o_{j i}$. being the $i$ th observed travel time on a road segment of category $j$ (and analogously for $c_{j i}$ ). The relative systematic bias is determined for each category $j$ separately as $\frac{\bar{c}_{j}-\bar{o}_{j}}{\bar{o}_{j}} \cdot 100 \%$.

\subsection{Results of the First Extension of the Approach}

For this analysis, the extended self-evaluation approach as described in Section 2.1 was applied on FCD for Berlin from October to December 2012 to assess the quality of the traffic data. The data pool consists of ten Wednesdays within this period, none of them being a public holiday in Germany.

Since the used algorithm also includes historic speeds as input for the computation of FCD results, similar to the observations in [4], significant changes in the systematic bias have been observed, which correspond to the change of traffic states in the course of a day (see Fig. 1). 

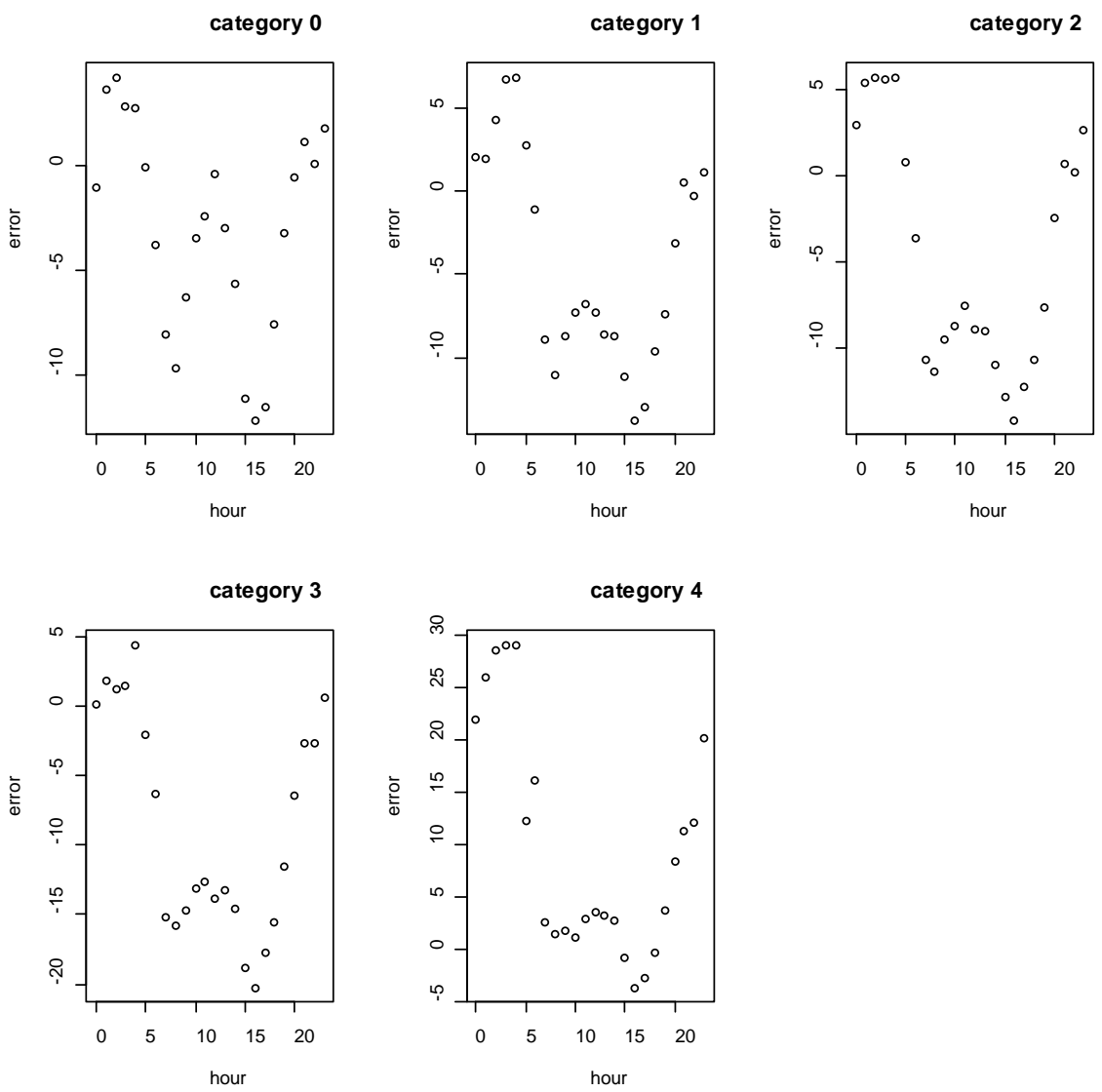

Figure 1. Systematic errors per street category and hour of day (results of experiments by DLR 2014).

For all categories the systematic error follows the typical daily course of speeds, where free flow at night and breakdowns during the rush hours in the morning and late afternoon can be observed. But only for category 0 between the morning and afternoon rush hours the same level of systematic error as for free flow intervals is reached again. All other categories have completely different systematic errors in the course of the whole day, and when comparing the free flow intervals during the night. Also for category 4, in contrast to other categories, travel times are greatly overestimated in comparison to trajectory travel times.

Potential reasons for differences in these results are category-specific traffic capacities or demands, but also category-specific speed limits which could have an influence if used as initial values for historic speeds. Since the adaption of traveling speed for a vehicle to the given speed limits often occurs at the transition between street categories as well as delay times due to right of way for roads of higher categories, the travel time decomposition is even more complicated for these cases 
and could also lead to systematic errors in the distribution of travel times between street categories (cf. [5]).

\section{Bias-Correction and Results}

This section gives the final approach to automated bias correction as a second extension of the original self-evaluation approach in [4].

The aim is an area-wide correction of the systematic bias on every link of the road map. It must be expected that the systematic error of the mean link travel times derived from the FCD system varies from one location (that is, from one section of road) to another. Due to the typically rather low penetration rates for PVD and the resulting lack of sufficient amounts of tracking data, it will often not be possible to do this separately for every individual link and for every period of interest. In other words, an area-wide bias correction would require an unrealistic amount of reference data covering all road sections.

For this reason, we propose a slightly different approach: with the assumption that the relative bias is fixed, i.e. the same for corresponding traffic situations, it can be transferred and used to correct the results of further computations.

More precisely: let $\hat{v}$ denote the "true" reference value for travel time on a link. Given that $\mu_{i} \neq 0$ for all of $n$ data sources $i=1, \ldots, n$ where $\hat{v}$ is the "true" expected value for data source $i$ during a particular time of day and on a particular road segment, we assume that $p_{i}:=\frac{\widehat{v}}{\mu_{i}}$ for all $i=1, \ldots, n$ is (more or less) constant for all considered roads. Then, each data source has a fixed relative error regarding its expectation $\mu_{i}=\mathbb{E}\left(X_{i}\right)$, where $X_{i}$ is the random variable for data source $i$. Notice that in contrast to the method of [4], this new approach aims at bias correction of $n$ data sources (e.g., FCD from $n$ vehicle fleets), which are now considered instead of only one. Therefore, the computation of systematic biases is done for each of the $n$ data sources separately.

The idea then is to estimate $p_{i}$ from a number of $m$ reference measurements (i.e. $p_{i}^{(j)}$ where $\left.j=1, \cdots, m\right)$ and to apply the sample mean $\overline{p_{i}}:=\frac{1}{m} \sum_{j=1}^{m} p_{i}^{(j)}$ with $i=1, \cdots, n$ again denoting the $n$ data sources, as a correction factor for area-wide online bias correction. For a practical implementation, and with the introductory remarks, this can only be done using the same relative systematic error for corresponding traffic situations.

Given that $p_{i}$ is a fixed number (i.e. $\overline{p_{i}}=p_{i}=\frac{\widehat{v}}{\mu_{i}}$ for all $i=1, \cdots, n$ ) by assumption, we formally obtain

$$
\mathbb{E}\left(p_{i} X_{i}\right)=\frac{\hat{v}}{\mu_{i}} \mathbb{E}\left(X_{i}\right)=\hat{v} .
$$


Hence, $Y_{i}:=\bar{p}_{i} X_{i}$ is an unbiased random measurement of the true value $\hat{v}$.

Next, we need to state in more detail

- $\quad$ how to estimate the sample means $\bar{p}_{i}$

- what precisely is meant by “corresponding traffic situations”.

Regarding the first point: analogously to the previous method of self-evaluation, for $i=1, \cdots, n$, percentage systematic biases are calculated as $\frac{\bar{c}-\bar{o}}{\bar{o}} \cdot 100 \%$ (cf. Section 2). Moreover, whereas the previous method was used for the diagnosis of the (global) systematic bias only, the present approach also aims at correcting any systematic bias in the data. For this purpose, correction factors $\bar{p}_{i}=\frac{\bar{o}_{i}}{\bar{c}_{i}}$ for every data source $i=$ $1, \cdots, n$ are calculated alongside the systematic biases. To see that the $\bar{p}_{i}$ with $i=1, \cdots, n$ as given here have in fact the property of correcting the systematic bias estimated from the $m$ sample trajectories for data source $i$, or more precisely, from the $m$ computed travel times $c_{i 1}, \ldots, c_{i m}$, and the $m$ observed actual travel times $o_{i 1}, \ldots, o_{i m}$, notice that in analogy to (1), we have that

$$
\frac{1}{m} \sum_{k=1}^{m} \bar{p}_{i} c_{i k}=\frac{\overline{p_{i}}}{m} \sum_{k=1}^{m} c_{i k}=\frac{\bar{o}_{i}}{\bar{c}_{i}} \cdot \overline{c_{i}}=\bar{o}_{i}
$$

For $i=1, \cdots, n$, the trajectory data of source $i$ is used for the calculation of $\bar{p}_{i}$. As has been said before, it will often not be possible to do this separately for every individual link and for every period of interest. Therefore, the approach followed here calculates the correction factors separately for each of $L$ sets of links with identical constructional attributes.

Regarding the second point, it also does this separately for each of $T$ sets of corresponding periods, respectively. Recall from Section 2.1 that, in order to extend the previous approach of self-evaluation to periodically computing systematic biases for each link of interest, a second assumption has to be made (besides the one that has already been made, i.e. assuming that actual travel times for individual vehicle trajectories can be used as a ground truth for the mean link travel times): it is assumed that it is possible to define corresponding periods (time slices) with typically similar traffic conditions. Based on this assumption, correction factors can be transferred from one (reference) period to corresponding periods.

More precisely, for $i=1, \cdots, n$ and each data source $i$, for $l=1, \cdots, L$ and each set $L_{l}$ of links with identical constructional attributes, and for $t=1, \cdots, T$ and each set $T_{t}$ of corresponding periods, a separate estimation of the true travel times $\hat{v}_{l t}$ on the links in $L_{l}$ for the periods in $T_{t}$ is done, using trajectory data of source $i$ (for an instructive example of corresponding periods see Figure 2). 


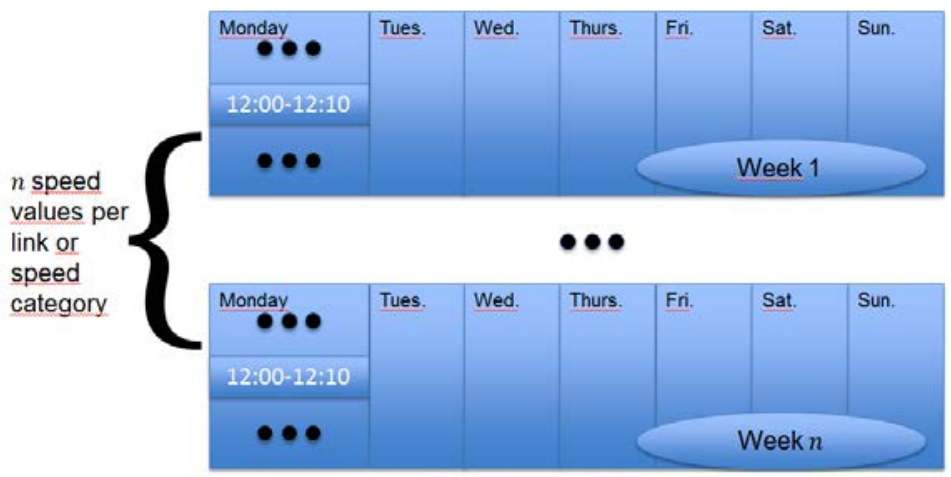

Figure 2: Example for corresponding periods (source: DLR 2014).

Thereby $\hat{v}_{l t}$ is estimated as the mean trajectory travel time $\bar{o}_{i l t}$ for trajectories of source $i$ on links in the particular set $L_{l}$, and during observation periods in $T_{t}$. In doing so, it is also assumed that the travel time on individual links along a trajectory can be determined without introducing a significant systematic bias. In other words, one assumes that a reintroduction of any significant systematic bias during the necessary arithmetic decomposition of the total trajectory travel time on individual links can be avoided by appropriate means (cf. [5]).

Then, separate estimations of the expected travel time $\mu_{i l t}$ for each data source $i$ and the aforementioned links and periods are done, as the mean travel time $\bar{c}_{i l t}$ on links of trajectories of data source $i$, which are also in the particular set $L_{l}$, and observed during periods in $T_{t}$, as computed by the FCD system, using tracking data from source $i$. The final correction factor used for $i=1, \cdots, n$ and each data source $i$, for $l=1, \cdots, L$ and each set $L_{l}$ of links with identical constructional attributes, and for $t=1, \cdots, T$ and each set $T_{t}$ of corresponding periods, is

$$
\bar{p}_{i l t}:=\frac{\bar{o}_{i l t}}{\bar{c}_{i l t}}
$$

This is an estimator for $\frac{\widehat{v}_{l t}}{\mu_{i l t}}$ (cf. our remark that $p_{i}$ is really fixed, right before Eq. (1)).

Notice that the approach only uses the trajectories of data source $i$ when calculating the estimator $\bar{o}_{i l t}$ for $\hat{v}_{l t}$. This is done in order to match the degree of data coverage on individual links during the computation of $\bar{c}_{i l t}$ for data source $i$, respectively. For this reason, there are $n$ estimators for the true travel times $\hat{v}_{l t}$, namely $\bar{o}_{i l t}$, one for every data source $i=1, \cdots, n$.

Then, finally, the $y_{i}$ with $i=1, \cdots, n$ are the unbiased measurements for data source $n$, that is, the bias-corrected realizations $y_{i}=\overline{p_{i}} x_{i}$ of the random variable $Y_{i}=\bar{p}_{i} X_{i}$ (indices $l$ and $t$ for the considered $L$ link sets and $T$ periods have been suppressed). The $x_{i}$ with $i=1, \cdots, n$ are the original biased measurements of data 
source $i$ (for the considered period set of interest, respectively). Thereby, choice of an appropriate correction factor $\overline{p_{i}}$ is based on the fact that a correspondence of periods at the same time of day (TOD) has been defined, as well as a correspondence of links with the same constructional attributes. In other words, the correction factor $\bar{p}_{\text {ilt }}$ for the $t$ th time slice and the $l$ th set of links, is used for every period corresponding to $t$ (i.e, for every period at the same TOD), and every link in the set of constructionally comparable links $L_{l}$.

Summarized, the approach followed here calculates the correction factors separately for each of $L$ sets of links with identical constructional attributes, and for each of $T$ sets of corresponding periods, respectively. Thereby it relies on the validity of the second assumption made (see above). It only remains to further illustrate the term "identical constructional attributes": To reduce the number of different cases that have to be considered and to be able to efficiently collect the needed reference data, traffic situations were categorized by the criteria used in Section 2.1, which had already yielded well-differentiated systematic biases.
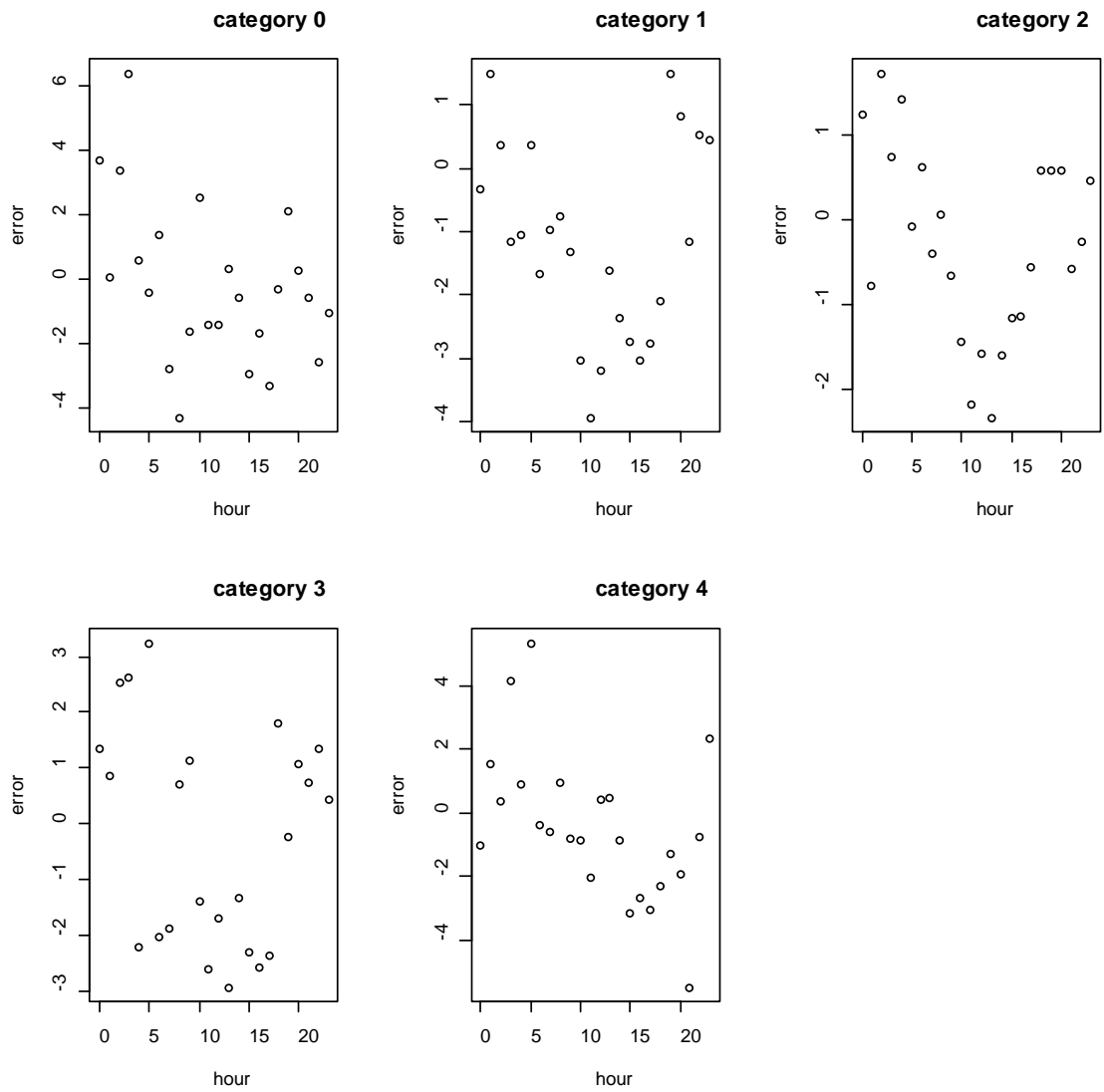

Fig. 3. Corrected FCD results by application of correction factors determined from a reference data pool (results of experiments by DLR 2014). 
To validate this approach, correction factors were determined for eight Wednesdays as one subset of the data pool, and then these factors were applied on the data for the remaining two Wednesday to test whether it is possible to effectively transfer the correction factors to other, corresponding time periods, and also if the selected separation criteria were good choices for the separation of traffic conditions.

These plots show that for the resulting corrected FCD (see Figure 3), the systematic bias was reduced dramatically in comparison with the uncorrected values (as presented before in Figure 1). Also the typical daily course of speeds is no longer visible and the average systematic bias per street category no longer deviates from zero as for the uncorrected results. This shows that the proposed assumption holds in this case and specific correction factors determined from the systematic bias of previous data can be applied to correct further results of FCD processing systems.

\section{Summary}

A new approach for automated bias-correction of traffic data has been developed, deployed and evaluated. Rather than basing the corrections on data from a few costly measurement campaigns, it bases its corrections on really large numbers of reference data which are not limited to specific parts of a road network. By combining data for similar traffic conditions, the required amount of reference data to generate correction factors can be collected within weeks. These factors are then applied automatically during online processing to generate corrected FCD results in real-time.

The clear dependence of the variance of the traffic data on the external factors that have been identified as separation criteria shows that these are indeed suitable to describe specific traffic conditions, and confirms the assumption that similar traffic conditions also result in similar systematic errors. While it cannot be expected that the systematic bias in FCD processing can be eliminated completely, e.g. due to small differences in traffic conditions between reference and application intervals, the results clearly show a significant overall reduction.

\section{Acknowledgment}

The research leading to these results has received funding from the European Union Seventh Framework Programme "SimpleFleet" (http://www.simplefleet.eu, grant agreement No. FP7-ICT-2011-SME-DCL-296423).

The authors thank the taxi control center Taxi Berlin TZB GmbH for provision of GPS-data for the taxi-FCD system. 


\section{References}

1. Brockfeld, E., Wagner, P., Passfeld, B.: Validating Travel Times calculated on the basis of taxi floating car data with test drives. In: 14th ITS World Congress. Beijing (2007)

2. Brockfeld, E., Sohr, A., Ebendt, R.: Validation of a taxi-FCD system by GPS - test drives. In: 17th World Conference on Intelligent Transport Systems. Busan (2010)

3. Reinthaler, M., Novotny, B., Hildebrandt, R., Weichenmeier, F.: Evaluation of speed estimation by floating car data within the research project DMotion. In: 14th ITS World Congress. Beijing (2007)

4. Kuhns, G., Ebendt, R., Wagner, P., Sohr, A., Brockfeld, E.: Self Evaluation of Floating Car Data Based on Travel Times from Actual Vehicle Trajectories. In: IEEE Forum on Integrated and Sustainable Transportation Systems. Vienna (2011)

5. Neumann, T.: Accuracy of distance-based travel time decomposition in probe vehicle systems, Journal of Advanced Transportation (2013) 